\title{
ASOCIACIÓN DE LA NEUROPATÍA AUTONÓMICA CARDIOVASCULAR Y EL INTERVALO QT PROLONGADO CON LA MORBIMORTALIDAD CARDIOVASCULAR EN PACIENTES CON DIABETES MELLITUS TIPO 2
}

\author{
Ray Ticse Aguirre ${ }^{1,2, a}$, Jaime E. Villena ${ }^{1,2, b, c}$
}

\begin{abstract}
RESUMEN
Con el objetivo de evaluar la relación entre la neuropatía autonómica cardiovascular (NACV) y el intervalo QT corregido (QTC) con la morbimortalidad cardiovascular en pacientes con diabetes mellitus tipo 2, se realizó el seguimiento a 5 años de 67 pacientes que acudieron a consulta externa del Servicio de Endocrinología. Se presentaron eventos cardiovasculares en 16 pacientes; el 82\% completó el seguimiento y se encontró que el intervalo QTc prolongado fue la única variable que se asoció de forma significativa a morbimortalidad cardiovascular en el análisis de regresión logística múltiple (RR: 13,56; IC 95\%: 2,01-91,36) ( $p=0,0074)$.
\end{abstract}

Palabras clave: Diabetes Mellitus Tipo 2, Neuropatías Diabéticas, Síndrome de QT Prolongado (fuente: DeCS BIREME)

\section{ASSOCIATION OF CARDIOVASCULAR AUTONOMIC NEUROPATHY AND PROLONGED QT INTERVAL WITH CARDIOVASCULAR MORBIDITY AND MORTALITY IN PATIENTS WITH TYPE 2 DIABETES MELLITUS}

\begin{abstract}
In order to evaluate the relationship between cardiovascular autonomic neuropathy and corrected QT interval (QTC) with cardiovascular morbidity and mortality in patients with type 2 diabetes mellitus, we followed up for 5 years 67 patients attending the outpatient Endocrinology Service. 82\% completed follow-up and cardiovascular events occurred in 16 patients. We found that long QTc interval was the only variable significantly associated with cardiovascular morbidity and mortality in the multiple logistic regression analysis (RR: $13.56,95 \% \mathrm{Cl}: 2.01-91.36)(p=0.0074)$.
\end{abstract}

Key words: Diabetes Mellitus Type 2, Diabetic Neuropathies, Long QT Syndrome (source: MeSH NLM).

\section{INTRODUCCIÓN}

En pacientes diabéticos, la prolongación del intervalo QTc se produce por enfermedad isquémica miocárdica y daño de fibras nerviosas del sistema autonómico cardiovascular. Estas complicaciones, sumadas a la nefropatía diabética, son las que más influyen en la morbilidad y mortalidad de estos pacientes ${ }^{(1)}$. En el Perú no existe ningún estudio de tipo cohorte sobre factores de riesgo para morbilidad y mortalidad cardiovascular; tampoco se ha evaluado el rol de la neuropatía autonómica y la prolongación del intervalo QTC en la sobrevida de pacientes con diabetes mellitus.

Con el propósito de conocer la importancia de la neuropatía autonómica cardiaca en la morbimortalidad de población peruana, se realizó un seguimiento por cinco años a un grupo de pacientes, donde se encontró que el factor más importante para la morbimortalidad cardiovascular fue la prolongación del intervalo QT corregido (QTC). Aunque se desconoce el significado clínico de la prolongación del intervalo QTc, se le asocia con arritmias y muerte súbita ${ }^{(2-5)}$.

\section{EL ESTUDIO}

Población de estudio. A partir de un estudio basal del año 2002, en el que se determinó la frecuencia de neuropatía autonómica cardiovascular en pacientes con diabetes mellitus tipo 2 que acudían regularmente al consultorio externo del Servicio de Endocrinología, se realizó el seguimiento a cinco años de 67 pacientes, entre 25 y 75 años de edad, enrolados por conveniencia. Los criterios de inclusión y exclusión se publicaron en el estudio basal ${ }^{(6)}$.

El seguimiento fue anual a través de la consulta externa y la revisión de la historia clínica.

Definición de neuropatía autonómica cardiovascular (NACV). Presencia de dos más respuestas anormales a las siguientes pruebas:

\footnotetext{
Departamento de Medicina. Hospital Nacional Cayetano Heredia.

2 Universidad Peruana Cayetano Heredia.

a Médico Internista; b Médico Endocrinólogo; c Profesor Principal de Medicina. Universidad Peruana Cayetano Heredia.
}

Recibido: 04-10-10 Aprobado: 09-03-11 
a. Variabilidad de la frecuencia cardiaca: con una frecuencia de cinco respiraciones/minuto se calculó el promedio de la frecuencia cardiaca más alta durante la inspiración y la más baja durante la espiración en tres ciclos consecutivos. Se consideró anormal si la diferencia entre los promedios fuera $\leq 10$ latidos $/ \mathrm{mi}-$ nuto.

b. Maniobra de Valsalva: cada paciente sopló mediante un conector acoplado a un tensiómetro de mercurio hasta $\geq 40 \mathrm{mmHg}$ durante 15 segundos. Se calculó el promedio del intervalo RR más largo después del procedimiento y el más corto durante el procedimiento, en tres ciclos consecutivos y luego se obtiene la razón. Se consideró anormal $\leq 1,10$.

c. Razón 30/15: con el paciente en posición supina se calculó el intervalo RR más largo alrededor del latido cardiaco 30 , luego el más corto alrededor del latido 15 en posición de pie. Se consideró anormal si la razón fue $\leq 1,00$.

d. Hipotensión ortostática: se consideró anormal la caída de la presión arterial sistólica > $20 \mathrm{mmHg}$, después de uno, tres y cinco minutos de pasar de la posición decúbito dorsal a la de pie.

Definición de morbimortalidad cardiaca. Desenlace compuesto de:

a. Evento cardiovascular no fatal: hospitalización por infarto del miocardio, síndrome isquémico coronario e insuficiencia cardiaca.

b. Evento cardiovascular fatal: muerte súbita o por infarto del miocardio o síndrome isquémico coronario.

Definición del Intervalo QT corregido. Valor obtenido mediante la siguiente formula: $Q T c=Q T /(R R)^{1 / 2}$. Se tomó el promedio de la medición de tres intervalos $\mathrm{QT}$ consecutivos y sus respectivos RR, obtenidos por registro electrocardiográfico, a velocidad de $50 \mathrm{~mm} / \mathrm{s}$ en segunda derivada. Se consideró prolongado un valor de QTc $>0,44 s$.

Procedimientos. Se registró la edad, sexo, tiempo de enfermedad (diabetes mellitus 2), índice de masa corporal, relación cintura/cadera, presión arterial sistólica, diastólica y media, creatinina, perfil de lípidos sanguíneos, glicemia en ayunas y hemoglobina glicosilada.

Las pruebas séricas de laboratorio fueron tomadas y procesadas en la Clínica Médica de la Universidad Peruana Cayetano Heredia. Las pruebas para neuropatía autonómica cardiaca y la medición del intervalo QTC fueron realizadas cada una por un solo evaluador, de manera independiente.
Aspectos éticos. El estudio basal fue aprobado por el comité de ética de la Universidad Peruana Cayetano Heredia ${ }^{(6)}$.

Análisis de datos. Para comparar proporciones se utilizó la prueba de Chi cuadrado considerando significativo $p<0,05$. Las variables presentaron distribución no normal por lo que se usó la prueba de Mann-Whitney. Se realizó el análisis de regresión logística múltiple entre las variables con significado estadístico para determinar las variables asociadas en forma independiente a la morbimortalidad cardiovascular. El análisis estadístico se realizó con el programa STATA 10.0 con asesoramiento del Departamento de Estadística de la Universidad Peruana Cayetano Heredia.

\section{HALLAZGOS}

El $82 \%$ (55 pacientes) completó el seguimiento. Seis pacientes no completaron el seguimiento por cambio domiciliario, seis pacientes por cambio de número telefónico y un paciente por negación a continuar participando. La comparación entre ambos grupos no reveló diferencias estadísticas respecto a la edad, tiempo de enfermedad, índice de masa corporal, presión arterial, frecuencia cardiaca y frecuencia de NACV (datos no mostrados).

Se presentaron eventos cardiovasculares en 16 pacientes: cinco fueron fatales (dos por accidente cerebrovascular; uno por infarto de miocardio y uno por muerte súbita) y 11 no fatales (seis con síndromes coronarios; dos con insuficiencia cardiaca y tres con enfermedad cerebrovascular).

En la Tabla 1 se muestra la comparación de las variables clínicas entre los pacientes con o sin morbimortalidad

Tabla 1.Comparación de las variables clínicas entre los pacientes con y sin morbi-mortalidad cardiovascular.

\begin{tabular}{|c|c|c|c|}
\hline & $\begin{array}{c}\text { Sin morbi-mor- } \\
\text { talidad cardio- } \\
\text { vascular }\end{array}$ & $\begin{array}{c}\text { Morbi-mortali- } \\
\text { dad cardiovas- } \\
\text { cular }\end{array}$ & $\mathbf{p}$ \\
\hline Número de pacientes & 39 & 16 & \\
\hline Sexo masculino (\%) & 80 & 30 & 0,51 \\
\hline Edad (años) & $55,97+/-9,05$ & $61,63+/-9.8$ & 0,045 \\
\hline $\begin{array}{l}\text { Tiempo de enfermedad } \\
\text { (años) }\end{array}$ & $7,25+/-6,49$ & $11,39+/-6,67$ & 0,037 \\
\hline Índice de masa corporal & $28,95+/-5,12$ & $28,64+/-4,25$ & 0,83 \\
\hline Relación cintura/cadera & $0,91+/-0,05$ & $0,92+1-0,049$ & 0,61 \\
\hline $\begin{array}{l}\text { Frecuencia cardiaca al } \\
\text { reposo }\end{array}$ & $76,84+/-13,53$ & $81,12+/-11,19$ & 0,26 \\
\hline Presión arterial sistólica & $122,07+/-16,4$ & $131,06+/-21,21$ & 0,09 \\
\hline Presión arterial diastólica & $70,89+/-7,4$ & $75,31+/-10,4$ & 0,8 \\
\hline $\begin{array}{l}\text { Variabilidad anormal de la } \\
\text { frecuencia cardiaca (\%) }\end{array}$ & 59,2 & 93,8 & 0,043 \\
\hline $\begin{array}{l}\text { Duración del intervalo } \\
\text { QTC (seg) }\end{array}$ & $0,41+/-0,034$ & $0,45+/-0,039$ & 0,00014 \\
\hline $\begin{array}{l}\text { Neuropatía autonómica } \\
\text { cardiaca (\%) }\end{array}$ & 35,9 & 81,3 & 0,0029 \\
\hline
\end{tabular}


Tabla 2. Comparación de las variables de laboratorio de los pacientes con y sin morbi-mortalidad cardiovascular.

\begin{tabular}{lccc}
\hline & $\begin{array}{c}\text { Sin morbi- } \\
\text { mortalidad } \\
\text { cardiovascular }\end{array}$ & $\begin{array}{c}\text { Con morbi- } \\
\text { mortalidad } \\
\text { cardiovascular }\end{array}$ & $\mathbf{p}$ \\
\hline Creatinina $(\mathrm{mg} / \mathrm{dl})$ & $0,9+/-0,18$ & $1,02+/-0,25$ & 0,105 \\
\hline Colesterol total $(\mathrm{mg} / \mathrm{dl})$ & $186,61+/-37,31$ & $204,45+/-37,34$ & 0,113 \\
HDL colesterol $(\mathrm{mg} / \mathrm{dl})$ & $42,68+/-8,64$ & $40,90+/-8,00$ & 0,485 \\
\hline LDL colesterol $(\mathrm{mg} / \mathrm{dl})$ & $111,02+/-37,47$ & $126,48+/-30,42$ & 0,15 \\
Triglicéridos $(\mathrm{mg} / \mathrm{dl})$ & $188,92+/-75,55$ & $178,27+/-55,31$ & 0,613 \\
Glucosa $(\mathrm{mg} / \mathrm{dl})$ & $142,05+/-55,82$ & $149,93+/-55,95$ & 0,636 \\
Hemoglobina & $9,67+/-2,28$ & $10,06+/-2,56$ & 0,581 \\
\hline glicosilada $\%)$ & & &
\end{tabular}

cardiovascular. No se encontró diferencias estadísticas en la comparación de variables de laboratorio (Tabla 2).

En el análisis de regresión logística múltiple se consideraron las variables que mostraron diferencias estadísticas en los análisis previos (edad, tiempo de enfermedad, intervalo RR, frecuencias de neuropatía autonómica cardiaca e intervalo QTc prolongado). Únicamente el intervalo QTc prolongado presentó riesgo relativo (RR) con valor estadístico relevante (Tabla 3 ).

\section{DISCUSIÓN}

Se conoce que la NACV incrementa la frecuencia de eventos cardiovasculares en pacientes con diabetes meIlitus tipo $2^{(7-10)}$ y que la frecuencia de esta enfermedad es elevada en pacientes peruanos. Durante el seguimiento a 5 años de 67 pacientes se descubrió que el factor más importante para morbimortalidad fue la prolongación del intervalo QT corregido. Tanto la NACV como la medición del intervalo QTc usualmente no son tomados en consideración en la evaluación de los pacientes.

El intervalo QT refleja la duración de la despolarización y repolarización del miocardio ventricular. Se desconoce la patogénesis de la prolongación de este intervalo, pero se sabe que en pacientes diabéticos tipo 2 está asociado con ciertos factores tales como: tiempo de enfermedad, cardiopatía isquémica, hipertensión arterial, insuficiencia renal y neuropatía autonómica cardiovascular (11-13). Es un fuerte predictor de eventos cardiovasculares mayores. Su asociación a la neuropatía autonómica cardiaca es tan alta que anteriormente se le consideró como un criterio diagnóstico ${ }^{(14-16)}$.

En el presente trabajo se encontró que la prolongación del intervalo QTC es predictor independiente de morbimortalidad. El hallazgo es controversial ya que es frecuente que se presente junto con otras complicaciones microvasculares como nefropatía y neuropatía. En la
Tabla 3. Riesgo relativo de morbi-mortalidad cardiovascular en pacientes con diabetes mellitus tipo 2. Resultado de la regresión logística.

\begin{tabular}{|c|c|c|c|}
\hline & $\mathbf{R R}$ & $\begin{array}{c}\text { Intervalo de } \\
\text { confianza } 95 \%\end{array}$ & $\mathbf{p}$ \\
\hline Edad & 1,05 & $(0,96-1,16)$ & 0,31 \\
\hline Tiempo de enfermedad & 1,05 & $(0,93-1,19)$ & 0,42 \\
\hline $\begin{array}{l}\text { Variabilidad anormal de la } \\
\text { frecuencia cardiaca }\end{array}$ & 3,59 & $(0,21-60,57)$ & 0,38 \\
\hline Neuropatía autonómica cardiaca & 1,67 & $(0,24-11,84)$ & 0,61 \\
\hline Intervalo QTc (>0,44 vs $\leq 0,44)$ & 13,56 & $(2,01-91,36)$ & 0,0074 \\
\hline
\end{tabular}

literatura científica se han encontrado otras investigaciones, realizadas con mayor número de pacientes y tiempo de seguimiento, que coinciden con los resultados presentados. En el estudio poblacional MONICA/ KORAAugsburg Cohort Study se realizó el seguimiento a nueve años de pacientes entre 55-74 años de edad, con y sin diabetes. Cuando se ajustaron los datos para factores cardiovasculares y variables demográficas, se encontró que la prolongación del intervalo QTc es un predictor independiente e incrementaba la mortalidad entre dos a tres veces en personas no diabéticas y diabéticas respectivamente. Esta asociación no fue hallada con la medida de la dispersión del intervalo QT ${ }^{(17,18)}$.

El estudio multicéntrico WHO Multinacional Study Of Vascular Disease, con 23 años de seguimiento, reveló que la prolongación del QTc se asocia con mortalidad en diabéticos tipo $1 \mathrm{y}$ el incremento de la frecuencia cardiaca al reposo en diabéticos tipo 2. Esto último refleja enfermedad de las vías nerviosas autonómicas cardiacas (19). En otra cohorte, de cinco años de seguimiento en pacientes con diabetes mellitus tipo 1 , se halló un riesgo relativo elevado (RR de 24,6$){ }^{(20)}$. La fuerza de asociación entre la prolongación del intervalo QTC y mortalidad se puede considerar entre las más altas reportadas en la literatura.

En el ensayo clínico ACCORD (Action to Control Cardiovascular Risk in Diabetes) se halló una alta mortalidad en el grupo de pacientes que estaban en control intensivo de la glicemia comparado con el grupo control (21). En un análisis posterior se evidenció que la prolongación del intervalo QTc actuó como un factor independiente de mortalidad ${ }^{22}$.

La investigación presenta la limitación de contar con un número reducido de pacientes y una cantidad importante de pacientes perdidos durante el seguimiento. También se considera una limitación el utilizar un desenlace cardiovascular compuesto, lo cual se usó justamente por el número pequeño de participantes. 
Los hallazgos de este trabajo sirven para mostrar que el uso de una prueba sencilla como es la medición del intervalo QT corregido, tiene un buen nivel de predicción de morbimortalidad cardiovascular. Se sugiere usarlo de forma rutinaria en la evaluación ambulatoria de los pacientes diabéticos.

\section{Fuentes de Financiamiento}

Hospital Nacional Cayetano Heredia y Universidad Peruana Cayetano Heredia.

\section{Conflictos de Interés}

Los autores declaran no tener conflictos de interés en la publicación del presente artículo.

\section{REFERENCIAS BIBLIOGRÁFICAS}

1. Veglio M, Sivieri R, Chinaglia A, Scaglione L, CavalloPerin P. QT interval prolongation and mortality in type 1 diabetic patients: a 5-year cohort prospective study. Neuropathy Study Group of the Italian Society of the Study of Diabetes, Piemonte Affiliate. Diabetes Care. 2000;23:1381-1383.

2. Vinik A, Ziegler D. Diabetic Cardiovascular Autonomic Neuropathy. Circulation. 2007;115:387-397.

3. Okin PM, Devereux RB, Lee ET, Galloway JM, Howard BV. Electrocardiographic repolarization complexity and abnormality predict all-cause and cardiovascular mortality in diabetes: the Strong Heart Study. Diabetes. 2004;53:434-440,

4. Salles GF, Bloch KV, Cardoso CR: Mortality and predictors of mortality in a cohort of Brazilian type 2 diabetic patients. Diabetes Care. 2004; 27:299-1305

5. Rossing P, Breum L, Major-Pedersen A, Sato A, Winding $\mathbf{H}$, Pietersen A, et al. Prolonged QTc interval predicts mortality in patie nts with type 1 diabetes mellitus. Diabet Med. 2001;18:199-205

6. Ticse R, Villena J, Pimentel R. Prevalencia de neuropatía autonómica cardiovascular en pacientes con diabetes mellitus tipo 2 de un hospital general. Rev Med Hered. 2007;18(3):129-135.

7. Ewing DJ, Martyn CN, Young RJ, Clarke BF. The value of cardiovascular function tests: 10 years experience in diabetes. Diabetes Care. 1985;8(5):491-8.

8. Orchard TJ, Lloyd CE, Maser RE, Kuller LH. Why does diabetic autonomic neuropathy predict IDDM mortality? An analysis from the Pittsburgh Epidemiology of Diabetes Complications Study. Diabetes Res Clin Pract. 1996;34Suppl1:S165-71.

9. Astrup AS, Tarnow L, Rossing P, Hansen BV, Hilsted J, Parving HH. Cardiac autonomic neuropathy predicts cardiovascular morbidity and mortality in type 1 diabetic patients with diabetic nephropathy. Diabetes Care. 2006;29(2):334-9.

10. Valensi P, Sachs RN, Harfouche B, Lormeau B, Paries J, Cosson E, et al. Predictive value of cardiac autonomic neuropathy in diabetic patients with or without silent myocardial ischemia. Diabetes Care. 2001;24:339-343.
11. Suys B, Heuten S, De Wolf D, Verherstraeten M, de Beeck LO, Matthys D, et al. Glycemia and corrected QT interval prolongation in young type 1 diabetic patients: what is the relation? Diabetes Care. 2006;29(2):427-9.

12. Veglio $M$, Chinaglia A, Cavallo-Perin P. QT interval, cardiovascular risk factors and risk of death in diabetes. J Endocrinol Invest. 2004;27(2):175-81.

13. Chiang CE, Roden DM. The long QT syndromes: genetic basis and clinical implications. J Am Coll Cardiol. 2000;36(1):1-12.

14. Rana BS, Lim PO, Naas AA, Ogston SA, Newton RW, Jung RT, et al. QT interval abnormalities are often present at diagnosis in diabetes and are better predictors of cardiac death than ankle brachial pressure index and autonomic function tests. Heart. 2005;91(1):44-50.

15. Benoit SR, Mendelsohn AB, Nourjah $P$, Staffa JA, Graham DJ. Risk factors for prolonged QTC among US adults: third National Health and Nutrition Examination Survey. Eur J Cardiovasc Prev Rehabil. 2005;12(4): 363-8.

16. Veglio M, Borra M, Stevens LK, Fuller JH, Perin PC. The relation between QTC interval prolongation and diabetic complications: the EURODIAB IDDM Complication Study Group. Diabetologia. 1999,42(1):68-75.

17. Beijers HJ, Ferreira I, Bravenboer B, Dekker JM, Nijpels G, Heine RJ, et al. Microalbuminuria and Cardiovascular Autonomic Dysfunction Are Independently Associated With Cardiovascular Mortality: Evidence for Distnct Pathways. Diabetes Care. 2009;32(9):1698-703.

18. Ziegler D, Zentai CP, Perz S, Rathmann W, Haastert B, Döring A, et al. Prediction of Mortality Using Measures of Cardiac Autonomic Dysfunction in the Diabetic and Nondiabetic Population: The MONICA/KORA Augsburg Cohort Study. Diabetes Care. 2008;31(3):556-61

19. Stettler C, Bearth A, Allemann S, Zwahlen M, Zanchin L, Deplazes M, et al. QTc interval and resting heart rate as long-term predictors of mortality in type 1 and type 2 diabetes mellitus: a 23-year follow-up. Diabetologia. 2007;50(1):186-94.

20. Veglio M, Sivieri R, Chinaglia A, Scaglione L, CavalloPerin P. QT Interval Prolongation and Mortality in Type 1 Diabetic Patients. A 5-year cohort prospective study. Diabetes Care. 2000;(9):1381-3

21. Gerstein HC, Miller ME, Byington RP, Goff DC, Jr., Bigger JT, Buse JB, et al. Effects of intensive glucose lowering in type 2 diabetes. N Engl J Med. 2008; 358:2545-59

22. Pop-Busui R, Evans G, Gerstein H, Fonseca V, Fleg $\mathbf{J}$, Hoogwerf B, et al. Effects of Cardiac Autonomic Dysfunction on Mortality Risk in the Action to Control Cardiovascular Risk in Diabetes (ACCORD) Trial. Diabetes Care. 2010;33(7):1578-1584.
Correspondencia: Ray Ticse Aguirre.

Dirección: Av. José Santos Chocano 1210, Urbanización Panamericana Norte, Los Olivos, Lima-Perú.

Correo electrónico: ray.ticse@upch.pe 